\title{
Microwave measurement of electron density and collision frequency of a pine fire
}

\author{
Kgakgamatso Mphale ${ }^{1,3}$ and Mal Heron ${ }^{2}$ \\ ${ }^{1}$ Physics Department, University of Botswana, P/Bag 0022. Gaborone, Botswana \\ ${ }^{2}$ Marine Geophysical Laboratory, James Cook Univeristy, Townsville, QLD 4811, Australia \\ E-mail:kgakgamatso.mphale@jcu.edu.au and mal.heron@jcu.edu.au
}

Received 1 November 2006, in final form 20 February 2007

Published 19 April 2007

Online at stacks.iop.org/JPhysD/40/2818

\begin{abstract}
Pinus caribea (pine) litter flame is a weakly ionized medium. Electron-neutral collisions are a dominant form of particle interaction in the flame. Assuming flame electrons to be in thermal equilibrium with neutrals and average electron-neutral collision frequency to be much higher than the plasma frequency, the propagation of microwaves through the flame is predicted to suffer signal intensity loss. A controlled fire burner was constructed where various natural vegetation species could be used as fuel. The burner was equipped with thermocouples and used as a cavity for microwaves with a laboratory quality network analyser to measure wave attenuation. Electron density and collision frequency were then calculated from the measured attenuation. The parameters are important for numerical prediction of electromagnetic wave propagation in wildfire environments. A controlled pine litter fire with a maximum flame temperature of $1080 \mathrm{~K}$ was set in the burner and microwaves $(8-10.5 \mathrm{GHz})$ were caused to propagate through the flame.

A microwave signal loss of 1.6-5.8 dB was measured within the frequency range. Based on the measured attenuation, electron density and electron-neutral collision frequency in pine fire were calculated to range from $0.51-1.35 \times 10^{16} \mathrm{~m}^{-3}$ and $3.43-5.97 \times 10^{10} \mathrm{~s}^{-1}$, respectively.
\end{abstract}

(Some figures in this article are in colour only in the electronic version)

\section{Introduction}

The absorption of energy by electrons in the flame may result in a considerable signal intensity loss for microwaves propagating through it. When the weakly ionized flame medium is illuminated with electromagnetic energy, electrons are accelerated by the electric field of the incident waves. Assuming that interaction between electrons and neutrals is elastic, the neutrals gain little kinetic energy during collisions mainly because they are relatively massive. Electrons are scattered isotropically such that the average velocity after collision is zero. In this way energy is transferred from the microwaves to the flame.

Weakly ionized gases at atmospheric pressure can effectively absorb microwaves. The absorption rate is directly related to $N_{\mathrm{e}} / v_{\text {eff }}$; where $N_{\mathrm{e}}$ is electron density and $v_{\text {eff }}$ is

3 Author to whom any correspondence should be addressed. momentum transfer collision frequency [1]. Several studies have taken advantage of the effect and determined ionization and momentum transfer collision frequency in flames and hot vapours, e.g. Smith and Sudden [11], Belcher and Sudden [12], Shuler and Weber [13] and Sturgeon et al [14]. Belcher and Sudden [12] studied the absorption of centimetric waves in a $1.45 \mathrm{~cm}$ wide alkali seeded coal gas-air flame at $2200 \mathrm{~K}$. From the absorption measurements, Belcher and Sudden [12] determined collision frequency and electron density to be $8.8 \times 10^{10} \mathrm{~s}^{-1}$ and $2.0 \times 10^{17} \mathrm{~m}^{-3}$, respectively. Smith and Sudden [11] carried out a very similar experiment but with hydrogen-air flames in a temperature range 19002000 K. Using electrodynamical theory, Smith and Sudden [11] calculated electron density in the range $(0.8-1.5) \times$ $10^{17} \mathrm{~m}^{-3}$ in the flames. Comparatively, clean unseeded flames contain lower ionization than seeded flames. For example, Adler [15] conducted an experiment in which radio waves 
were caused to propagate through pure jet fuel flames at a temperature of $1920 \mathrm{~K}$. Using propagation theory, ionization and collision frequency of $1.9 \times 10^{12} \mathrm{~m}^{-3}$ and $6.5 \times 10^{8} \mathrm{~s}^{-1}$, respectively were observed in [15]. Shuler and Weber [13] also performed a microwave propagation experiment on unseeded lean hydrogen-oxygen flames at adiabatic temperatures which ranged from 2400 to $3000 \mathrm{~K}$. Electron density in the hydrogenoxygen flames ranged from 2.5 to $3 \times 10^{15} \mathrm{~m}^{-3}$ and average momentum transfer collision frequency was determined to be $2.6 \times 10^{11} \mathrm{~s}^{-1}$.

Based on studies by [1,2,11-14], microwave communication efficiency on wildfire grounds, where adiabatic flame temperatures could be up to $1900^{\circ} \mathrm{C}$, may be impaired by the weakly ionized environment. Strong microwave attenuation is anticipated to occur at very hot regions of the fire [3,4]. Remote sensing is now used to detect surface and subsurface wildfires $[16,17]$. Active remote sensing devices such as radar at microwave frequencies are able to detect large scale fires. However, for very high intensity wildfires $\left(90 \mathrm{MW} \mathrm{m}^{-1}\right)$ with electron densities up to $10^{18} \mathrm{~m}^{-3}$, dielectric constants are low since they are highly conductive. This implies that the intensity of radar received signals may be very low.

Propagation tests have been carried out in wildfire environments. In the experiments, signals were caused to propagate above fire. As a consequence, no significant attenuation was observed. However, at flame electron densities and collision frequencies cited in the literature, surface and subsurface fires could be remotely detected because of low attenuation constant at wavelengths of $8-12 \mu \mathrm{m}$. The experiment investigates microwave propagation at a very hot and weakly ionized region of a pine fire with emissive power of $62 \mathrm{~kW} \mathrm{~m}^{-2}$. Signal attenuation was measured using a 2-port vector network analyser (VNA). Flame electron density and electron-neutral collision frequency were then calculated from the scattering parameter (s-parameter) determined attenuation.

\section{Ionization in the fire}

Oxidation reactions in the combustion zone of the fire are exothermic in nature. Fierce temperatures produced in the reaction zone, which could be up to $1900^{\circ} \mathrm{C}$, thermally excite the flame particulates. The excited particles can then be ionized to produce electrons and ions on a selective basis determined by temperature and ionization potential. Vegetation contains the element potassium in significant amounts. It can be up to $3.4 \%$ of a plant's dry weight [18]. The species can exist; ionically or organically attached to oxygen-containing functional groups (i.e. $-\mathrm{O}-\mathrm{C}-$ ) of the organic structure of plants, as discrete particles in voids of the organic matrix and in solution form such as in xylem vessels. During combustion, the potassium species are released from a thermally crumbling plant structure and convectively drawn into the combustion zone of the fire whereupon thermal decomposition ionize according to the equation:

$$
\mathrm{K}(g) \Leftrightarrow \mathrm{K}^{+}(g)+\mathrm{e}^{-} .
$$

The presence of hydrogen and methyl radical in pyrolyzing vegetative material promotes the release of potassium into the combustion zone as atoms [19]. Apart from potassium, other species that could appreciably thermally ionize are sodium $(\mathrm{Na})$ and graphitic carbon $\left(\mathrm{C}_{n}\right)$. Sodium has an ionization potential of $5.1 \mathrm{eV}$ while that of graphitic carbon or soot could be as low as $8.5 \mathrm{eV}$ [13]. Potassium stands out as the most favoured source of thermal ions in the combustion zone because of its abundance and low ionization potential $(4.34 \mathrm{eV})$.

Chemi-ionization is another possible mechanism by which significant ionization may occur in wildfire flames [6]. In the process, dissociation reactions provide part of the energy required for ionization since they are exothermic and the rest is from the flame. After the combustion of vegetative matter, alkaline earth metal oxide and carbonates settle as ash. There is a high possibility that soon after their formation in the flame, they react with one of the main products, carbon monoxide (CO), to produce electrons and carbon dioxide $\left(\mathrm{CO}_{2}\right)$ (see [20]). An example of the dissociation reaction is when an alkaline earth metal oxide such as calcium oxide $(\mathrm{CaO})$ reacts with $\mathrm{CO}$. The reaction may proceed in the following manner:

$$
\mathrm{CaO}+\mathrm{CO} \Leftrightarrow \mathrm{Ca}^{+}+\mathrm{e}^{-}+\mathrm{CO}_{2} .
$$

During the plant matter char combustion, freed potassium salts thermally dissociate into atoms. However not all thermally ionize, some dissociated potassium atoms may also react in the following manner to give electrons to the hot gaseous medium:

$$
\mathrm{K}+\mathrm{CO}+\mathrm{O} \Leftrightarrow \mathrm{K}^{+}+\mathrm{e}^{-}+\mathrm{CO}_{2}
$$

Vegetation species contain up to $1.1 \%$ chlorine on a dry mass basis. Chlorine exists in soluble form mostly attached to alkali atoms, e.g. $\mathrm{KCl}$ [21]. After thermal decomposition of the alkali chloride, chlorine ions in the flame may also contribute to the ionization by reacting with hydrogen atoms, also present in the flame, to give electrons according to the following equation:

$$
\mathrm{Cl}^{-}+\mathrm{H} \Leftrightarrow \mathrm{e}^{-}+\mathrm{HCl}
$$

Excited methyl radical $\mathrm{CH}^{*}$ is a known contributor to chemi-ionization in flames, e.g. in [5]. The $\mathrm{CH}$ radical reacts with oxygen atoms in the flame to produce $\mathrm{CHO}^{+}$, a primary ion in hydrocarbon flames [7], and electrons according to the following reaction equation:

$$
\mathrm{CH}^{*}+\mathrm{O} \Leftrightarrow \mathrm{CHO}^{+}+\mathrm{e}^{-} .
$$

\section{Flame propagation constant}

The propagation constant $(\gamma)$ of a dielectric medium such as a weakly ionized flame is given by the following relation:

$$
\gamma=\alpha_{\mathrm{f}}+\mathrm{i} \beta_{\mathrm{f}},
$$

where $\alpha_{\mathrm{f}}$ and $\beta_{\mathrm{f}}$ are attenuation and phase constants. When xband microwaves illuminate weakly ionized, highly collisional atmospheric pressure flame plasma, $\alpha_{\mathrm{f}}$ and $\beta_{\mathrm{f}}$ are related to electron-neutral collision frequency $\left(\varphi_{\text {eff }}\right)$ and ionization by the expressions:

$$
\alpha_{\mathrm{f}} \cong \frac{\varphi_{\mathrm{eff}}}{2 c}\left[\frac{\omega_{\mathrm{p}}^{2}}{\left(\omega^{2}+\varphi_{\text {eff }}^{2}\right)}\right]
$$

and

$$
\beta_{\mathrm{f}} \cong \frac{\omega}{c}\left[1+\frac{\omega_{\mathrm{p}}^{4}}{8\left(\omega^{2}+\varphi_{\mathrm{eff}}^{2}\right)^{2}} \frac{\varphi_{\mathrm{eff}}^{2}}{\omega^{2}}\right],
$$

where $\omega$ and $c$ are propagation cyclic frequency and speed of light in vacuum respectively. 


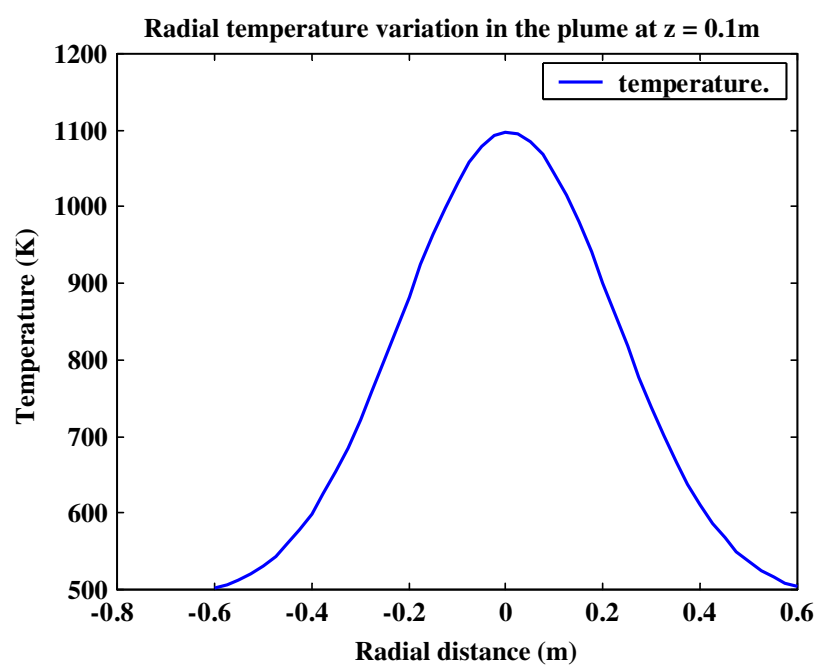

Figure 1. Fire temperature across the circular plume.

\section{Prediction of spatial variation of electron density and collision frequency in a circular fire plume}

\subsection{Prediction of flame temperature}

Theoretically, temperature in a circular fire plume is a function of radial distance $(r)$ from the seat of the fire. Dupuy et al [22] observed that temperature in the flame $\left(T_{\mathrm{sf}}\right)$ of such a fire varies as

$$
T_{\mathrm{f}}=T_{\mathrm{a}}+C \exp \left(-\varsigma\left(\frac{r}{z}\right)^{2}\right),
$$

where $C$ and $\zeta$ are empirical constants. $T_{\mathrm{a}}$ is ambient air temperature; $r$ and $z$ are radial distance from the seat of the fire and height above the combusting fuel, respectively. A typical curve for the variation of plume temperature with radial distance is shown in figure 1. It is a Gaussian profile where temperature decreases exponentially from a maximum value at the seat of the fire (i.e. at $r=0$ ) to a low value at the edges of the plume.

Along the axis of the plume, temperature decreases exponentially with height $(z)$. For fire with a seat temperature of about $1085 \mathrm{~K}$ and a flame height of about $1.0 \mathrm{~m}$ as considered in the experiment, its axial temperature may decrease to $580 \mathrm{~K}$ at about $1.2 \mathrm{~m}$ (see figure 2). At a point which is a perpendicular distance of $15 \mathrm{~cm}$ away from the seat of the fire, temperatures are predicted from equation (9) to be slightly lower than along the axis of the plumes. The equation predicts a maximum temperature of $1015 \mathrm{~K}$ at $z=0.1 \mathrm{~m}$ which decreases to $510 \mathrm{~K}$ at $1.2 \mathrm{~m}$ above the vegetation fuel (figure 2).

\subsection{Estimation of electron density}

As discussed in section 2, flame electrons are assumed to be produced mainly from the thermal ionization of potassium atoms which are produced from radial potassium complex reduction reaction. The electron density $\left(N_{\mathrm{e}}\right)$ can be estimated from an expression given by Frost [23] as

$$
N_{\mathrm{e}}=\left(K_{\mathrm{I}} N_{\mathrm{p}}\right)^{1 / 2}\left[\left(1+\frac{K_{\mathrm{I}}}{4 N_{\mathrm{p}}}\right)^{1 / 2}-\left(\frac{K_{\mathrm{I}}}{4 N_{\mathrm{p}}}\right)^{1 / 2}\right],
$$

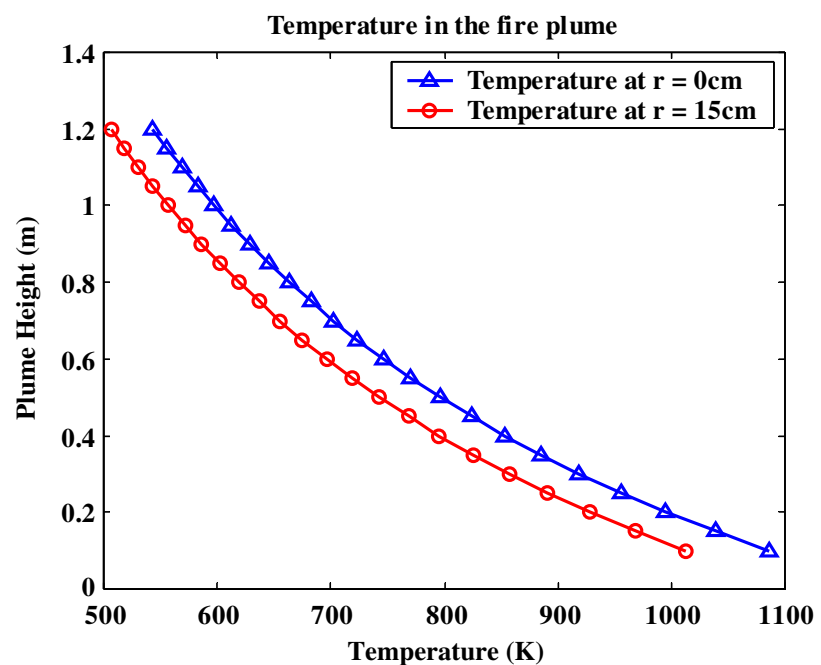

Figure 2. Flame temperatures variation with plume height.

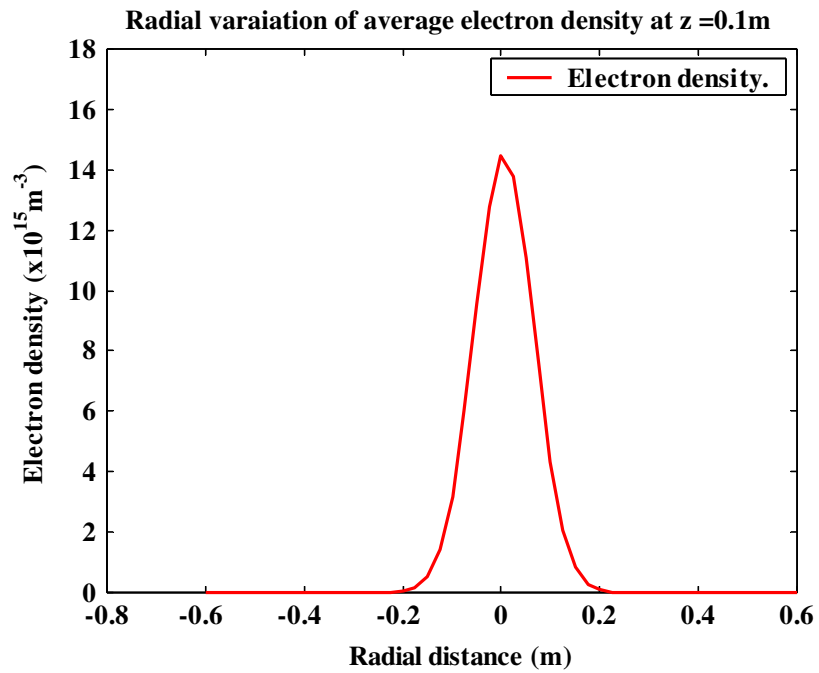

Figure 3. Radial electron density profile for a Gaussian temperature profile with maximum temperature of $1100 \mathrm{~K}$.

where $K_{I}$ is ionization equilibrium constant and $N_{\mathrm{p}}$ is the total number of ionized potassium particles given by

$$
N_{\mathrm{p}}=7.335 \times 10^{27} \mu \mathrm{T}^{-1} \mathrm{~m}^{-3} .
$$

Mphale and Heron [24] give an account of how to estimate the number of potassium atoms that could be available for ionization $(\mu)$ in a vegetation fire. The ionization equilibrium constant for ionization $\left(K_{\mathrm{I}}\right)$ is given by Saha's equation as

$$
K_{\mathrm{I}}=\frac{2 P_{\mathrm{m}^{+} \mathrm{int}}}{P_{\mathrm{mint}}}\left(\frac{2 \pi \cdot M_{\mathrm{e}} k T}{h^{2}}\right)^{3 / 2} \exp \left(\frac{-\left(E_{\mathrm{i}}\right)}{k T}\right),
$$

where $P_{\text {mint }}$ and $P_{\mathrm{m}^{+} \text {int }}$ are internal partition functions for potassium particles which are 2 and 1 at combustion temperatures $\left(1000^{\circ} \mathrm{C}\right)$, respectively. $E_{\mathrm{i}}, M_{\mathrm{e}}, k$ and $h$ are ionization energy, mass of an electron, Boltzmann and Plank constants, respectively. Application of equations (10)-(12) gives a radial electron density profile given in figure 3 for the temperature profile in figure 1 when vegetation is assumed to contain $0.5 \%$ potassium and up to $1 \%$ of it volatilizes into the combustion zone of the plume (see [24]). 


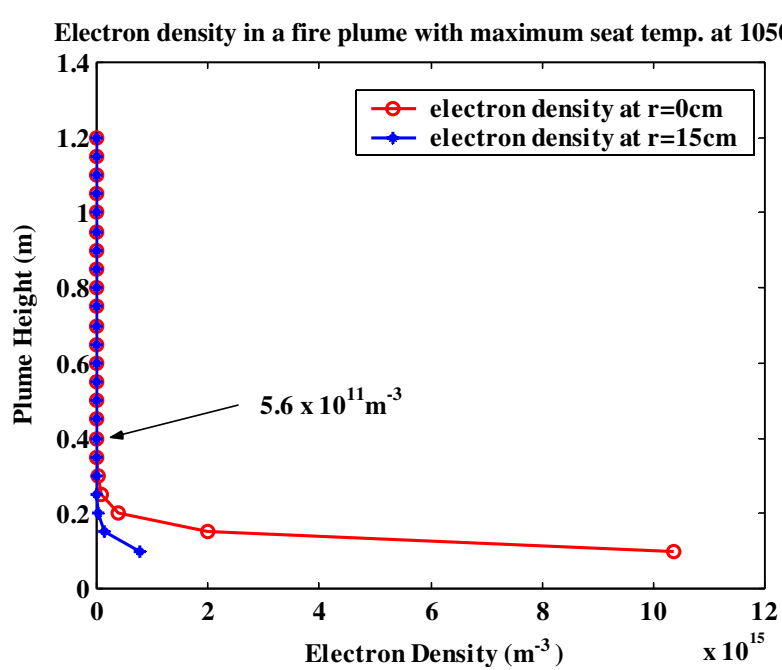

Figure 4. Variation of electron density with plume height at $r=0$ and $0.15 \mathrm{~m}$.

The influence of temperature is noticeable when the variation of electron density with height above the fuel surface is considered for two radial positions. Along the axis of the fire $(r=0)$, electron density is predicted to decrease exponentially with height from a maximum $1.4 \times 10^{16} \mathrm{~m}^{-3}$ to a comparatively very low value at $1.2 \mathrm{~m}$. At $r=0.15 \mathrm{~m}$ electron density decreases from $7.8 \times 10^{14} \mathrm{~m}^{-3}$ to $5.6 \times 10^{11} \mathrm{~m}^{-3}$ at $40 \mathrm{~cm}$ above the fuel surface (see figure 4 ).

\subsection{Estimation collision frequency}

Momentum transfer electron-neutral collision $\left(\varphi_{\text {eff }}\right)$ can be modelled from a relation given by Vyskocil and Musil [25] as

$$
\varphi_{\text {eff }}=7.33 \times 10^{3} N_{\mathrm{m}} a^{2} \sqrt{T},
$$

where $T$ is temperature, $N_{\mathrm{m}}$ number density of air molecules and $a$ is the radius of an air molecule. It is clear from the relation that electron collision frequency is dependent on flame temperature and the presence of neutral gas in the combustion area. At $1.2 \mathrm{~m}$ above the fuel surface, there is sufficient neutral gas to maintain electron-neutral collision frequency at about $1 \times 10^{11} \mathrm{~s}^{-1}$ (figure 5). At the combustion zone neutral gas density is insufficient to sustain the collision frequency at $1 \times 10^{11} \mathrm{~s}^{-1}$. However, insufficient ambient air and high temperature in the combustion zone lower the collision frequency values as low as $3 \times 10^{10} \mathrm{~s}^{-1}$ (figure 5).

\section{Experimental set-up}

\subsection{VNA system and combustion fuel}

The equipment used to measure the microwave absorption by the flames consists of a hexagonally shaped burner with an insulated wooden casing, a Hewlett-Packard 8577C VNA with $\mathrm{x}$-band horns and a computer. On the inside of the burner, a thick ( $8 \mathrm{~cm}$ wide) thermally insulating material known as Fiberfrax ${ }^{\circledR}$ was used to protect wood from the fire and heat. The material was lined to form a combustion area that is circular in base cross section. Two vent holes of $25 \mathrm{~mm}$ diameter were drilled on each of the sides, except the ones with horn inlets,

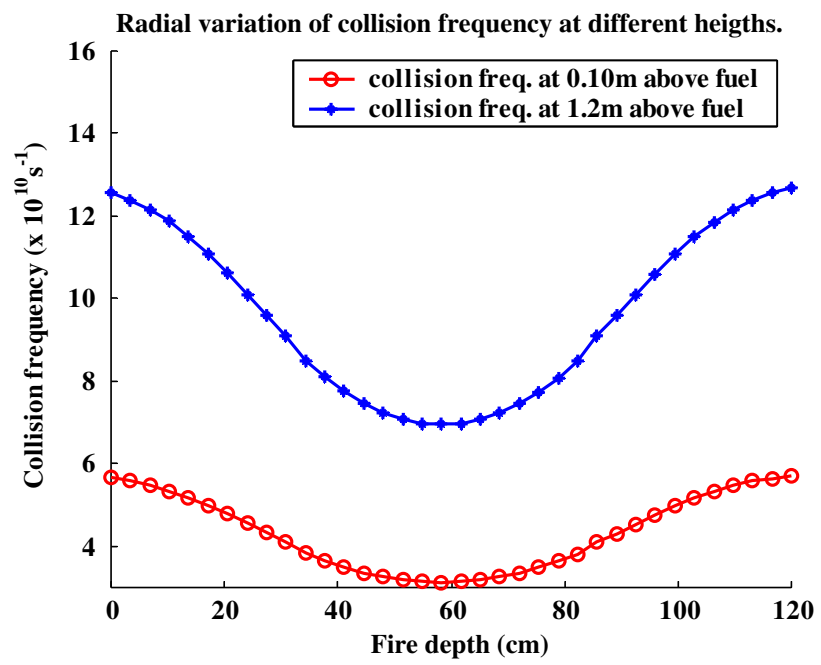

Figure 5. Estimation of electron-neutral collision frequency at $z=0.1$ and $120 \mathrm{~cm}$.

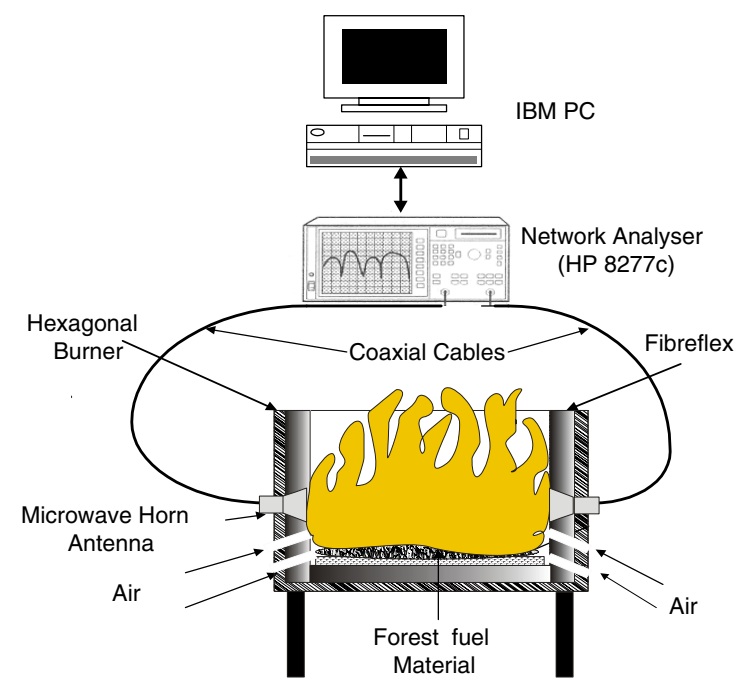

Figure 6. VNA set-up for $S_{21}$ and $S_{11}$ parameter measurements.

to allow air to enter and mix with fuel during combustion. Two holes of horn dimensions were also cut out from the burner casing directly opposite to each other and wooden supports were provided to secure the horns firmly to the wooden casing. The internal diameter of the burner was lined with Fiberfrax ${ }^{\circledR}$ which was set to $50 \mathrm{~cm}$. Adding to or slightly reducing the insulating material varied the propagation path length. Two x-band transmit-receive horns were used in the experiment. They were connected to the VNA through the twoport s-parameter test set by coaxial cables. High quality mode transition adapters were used to make the connections between coaxial cables and the horns. The set-up is shown in figure 6 .

Pine litter of bulk density of $4.92 \mathrm{~kg} \mathrm{~m}^{-3}$ was collected ten days before the experiment and left to dry in a laboratory to maximize combustion efficiency during burning.

\subsection{Flame temperature measurement}

A thermocouple tree about $1.25 \mathrm{~m}$ high was constructed from a steel pipe of diameter $0.025 \mathrm{~m}$. Side 'arms' of length $0.4 \mathrm{~m}$ 
were attached at every $0.25 \mathrm{~m}$ of the tree's height to hold at least four thermocouples. The thermocouples used were cut from a $100 \mathrm{~m}$ double braided fibreglass insulated chromelalumel $(24-\mathrm{G} / \mathrm{G})$ thermocouple wire $50 \mu \mathrm{m}$ in diameter. The thermocouple wire had a fibreglass shield which can withstand temperatures up to $450{ }^{\circ} \mathrm{C}$. The type $\mathrm{K}$ thermocouple wires were then electro-fused at one end to make a perfect junction and then tested with a hot air gun and a multimeter. The thermocouples were then fixed to tree 'arms' by means of a muffler tape and the electro-fused junctions were left protruding $1 \mathrm{~cm}$ beyond the 'arm' length into the flame. The thermocouples were then wired to a PICO ${ }^{\circledR}$ Tech TC-O8 data logger to read the temperatures into a computer throughout each experiment.

\subsection{S-parameter measurements}

The $8577 \mathrm{C}$ VNA set is designed to sweep from $50 \mathrm{MHz}$ to $40 \mathrm{GHz}$ and $\log$ in $601 \mathrm{~s}$-parameter data points in each and every sweep. The data can then be uploaded and analysed by the computer. The analyser takes $2 \mathrm{~s}$ to sample over one sweep, and then there is a latency of about $50 \mathrm{~s}$ before the next sweep can be initiated. Before the VNA was used it was calibrated first. The calibration method used in the experiment was the transmit-reflect-line (TRL). Varadan et al [8] give a full account of calibrating a network analyser using the TRL method. However, for the experiment, the frequency range of interest was between 8 and $10.5 \mathrm{GHz}$. Several sweeps and logging of s-parameters were carried out, but those for which flames filled the entire internal hollow space of the burner were chosen for s-parameter analysis. The selected logged in s-parameters were those at AS1, AS2, AS3 and AS4, which corresponded to the times 72, 135, 217 and $274 \mathrm{~s}$ after ignition.

\subsection{Determination of propagation constant from s-parameter}

The VNA measures the scattering parameters (s-parameters) from which the propagation constant can be calculated. From the s-parameters analysis, the propagation constant is related to the propagation factor $(T)$ by the relation

$$
\gamma=[\ln (1 / T)] / d,
$$

where $d$ is the length of the path traversed by the electromagnetic beam through the flame. s-parameters $\left(S_{11}\right.$ and $S_{21}$ ) determined from the VNA are then used to calculate the propagation factor using the relation [28]

$$
T=\left[\frac{S_{11}+S_{21}-\Gamma}{1-\left(S_{11}+S_{21}\right) \Gamma}\right],
$$

where $\Gamma$ is the reflection coefficient, which is given by the relation [28]

$$
\Gamma=\Omega \pm \sqrt{\Omega^{2}-1}
$$

where $\Omega=\left(\frac{S_{11}^{2}-S_{21}^{2}+1}{2 S_{11}}\right)$. The sign in (16) is chosen so that $|\Gamma|<1$. With $T$ determined from s-parameter using the network analyser, the propagation constant $\gamma$ can be worked out from (14). Then $\alpha_{\mathrm{f}}$ and $\beta_{\mathrm{f}}$ are determined from (16) as they are the real and imaginary parts of the propagation constant $(\gamma)$.
5.5. Determination of collision frequency and electron density

The inverse of the real part of the propagation constant determined from s-parameters is used graphically to determine collision frequency and electron density of the pine flame between the times AS1 and AS4 after ignition.

5.5.1. Collision frequency. Inverting equation (7) gives;

$$
\frac{1}{\alpha_{\mathrm{f}}}=\frac{\varsigma\left(\omega^{2}+\varphi_{\mathrm{eff}}^{2}\right)}{\varphi_{\mathrm{eff}}},
$$

where $\varsigma=\frac{2 c}{\omega_{\mathrm{p}}^{2}} ; \omega_{\mathrm{p}}^{2}=\left(\frac{N \mathrm{e}^{2}}{m \varepsilon_{0}}\right)$ and $\varepsilon_{0}$ are the plasma collision frequency and free space permittivity. A plot of $\frac{1}{\alpha_{\mathrm{f}}}$ versus $\omega^{2}$ gives a linear function where $\left(\varsigma / \varphi_{\text {eff }}\right)$ is the gradient (grad.) and $\varsigma \varphi_{\text {eff }}$ is the ordinate (ord.) at zero abscissa of the graph. Electron-neutral collision frequency $\left(\varphi_{\text {eff }}\right)$ is calculated from grad. and ord. as

$$
\varphi_{\mathrm{eff}}=\left(\frac{\text { ord. }}{\text { grad. }}\right)^{1 / 2} .
$$

5.5.2. Electron density. Electron density in the flame is calculated from the relation

$$
N=\left(\frac{2 c m . \varepsilon_{0}}{q_{\mathrm{e}}^{2}}\right) \frac{1}{\sqrt{\text { grad. } \times \text { ord. }}}
$$

\section{Experimental results and discussions}

\subsection{Flame temperatures}

Flames up to $100 \mathrm{~cm}$ high were observed during the experiment as air entrained through the holes at the bottom of the hexagonal burner to facilitate continuous combustion. The flames due to the combustion of pine litter took up to $6 \mathrm{~min}$ to extinguish. It took $10 \mathrm{~s}$ for flames to fill the inner space of the burner. Fire plume temperatures at different heights were logged in but those important for the experiment were those from the pine litter surface. Pine litter surface temperatures during the experiment are shown in figure 7 . The litter surface temperature rose rapidly to reach a maximum of $1081 \mathrm{~K}$ in $76 \mathrm{~s}$. This was $4 \mathrm{~s}$ after the first set of s-parameters (AS1) were logged in. At AS1, the temperature of the litter surface was $1075 \mathrm{~K}$. After reaching the climax, the surface temperature dropped steeply, though not as quickly as it rose from ignition, to AS2, a second log in point where the pine litter flame filled the inner hollow of the burner. AS2 is $135 \mathrm{~s}$ from ignition. At AS2, the pine litter surface temperature was observed to be $878 \mathrm{~K}$.

Several s-parameters were logged in after AS2, but those selected for analysis were logged in at AS3 and AS4, corresponding to 217 and $274 \mathrm{~s}$ after ignition, respectively. Surface temperatures at AS3 and AS4 are 728 and $646 \mathrm{~K}$, respectively. 


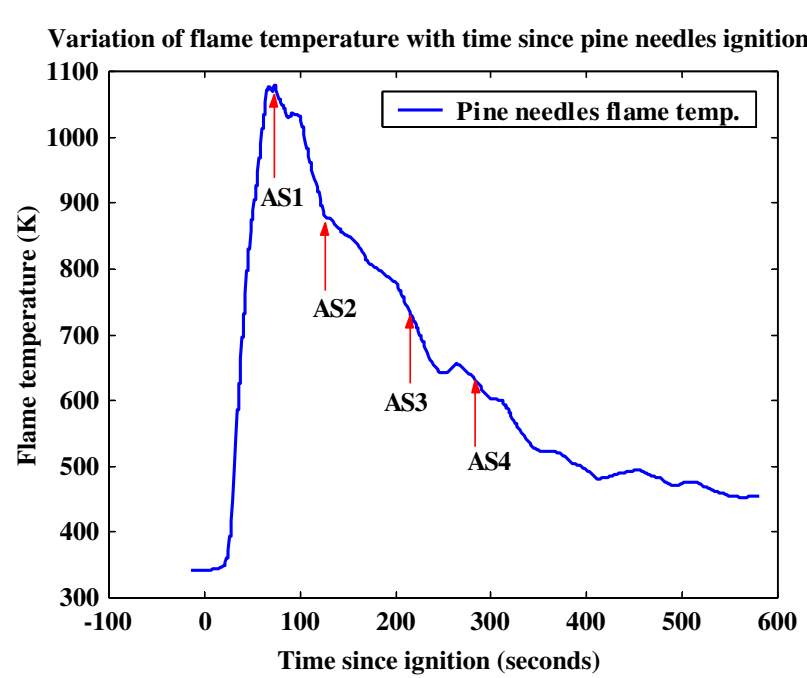

Figure 7. Pine litter surface fire temperature during the experiment.

\subsection{Microwave attenuation}

Microwave propagation through the hottest part of the flames suffered a significant signal intensity loss. Before the fuel in the burner was set alight (no flame condition), the VNAburner system was checked for a systematic signal loss. An average systematic loss of the VNA-burner system was found to be $7.2 \mathrm{~dB}$. Attenuation measurements at AS1 to AS3 for microwave frequencies $(8-10.5 \mathrm{GHz})$ are shown in figure 8 . The flame at AS1 was the most intense and it caused a signal loss of 5.68-3.70 dB for the frequency range, respectively. At AS2, 8-10 GHz microwaves incurred a signal loss of 3.17$1.94 \mathrm{~dB}$, respectively, while the cooler flame at AS3 imposed a signal intensity loss of $2.38-1.64 \mathrm{~dB}$ for the frequency range, respectively. As can be observed from figure 8, flame temperature plays a significant role in microwave absorption in the burner. A very hot flame imposes strong microwave absorption. High temperatures cause a significant ionization of incumbent flame particles. The effect could be amplified by the presence of alkali species in the flame, which is true for the combustion of vegetative matter. Plant matter contains up to $3.4 \%$ element potassium on a dry weight basis. During combustion of plant matter, potassium species are released from a thermally crumbling plant structure and convectively drawn into the combustion zone of the fire. High temperature causes thermal dissociation of the potassium species as they have low dissociation energies. Vodacek et al [9] estimate that $10-20 \%$ of potassium in vegetation is ionized in fires. This makes vegetation fires a weakly ionized environment with the capability to cause significant microwave absorption at the most intense regions of the fire.

\subsection{Electron density and collision frequency during flaming}

At AS1, the gradient of the plot was $4.09 \times 10^{-22}$ (see figure 9) and the ordinate was observed to be $4.81 \times 10^{-1}$. The gradient and ordinate values gave electron density and collision frequency for the pine litter flame to be $1.35 \times 10^{16} \mathrm{~m}^{-3}$ and $3.43 \times 10^{10} \mathrm{~s}^{-1}$.

At AS2, the gradient and the ordinate for the pine litter flame were $7.25 \times 10^{-22}$ and $9.20 \times 10^{-1}$. The gradient

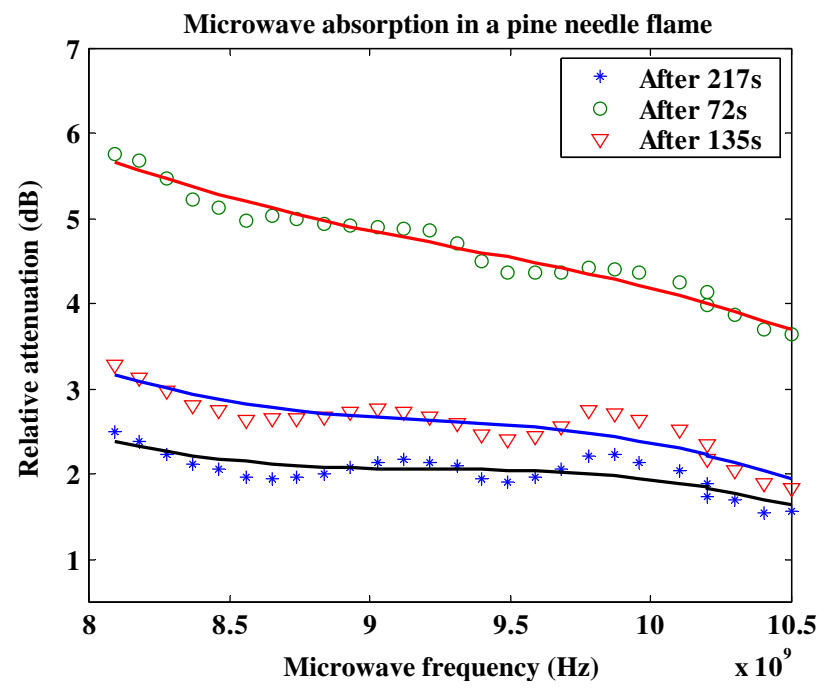

Figure 8. Variation of relative attenuation with propagation frequency.

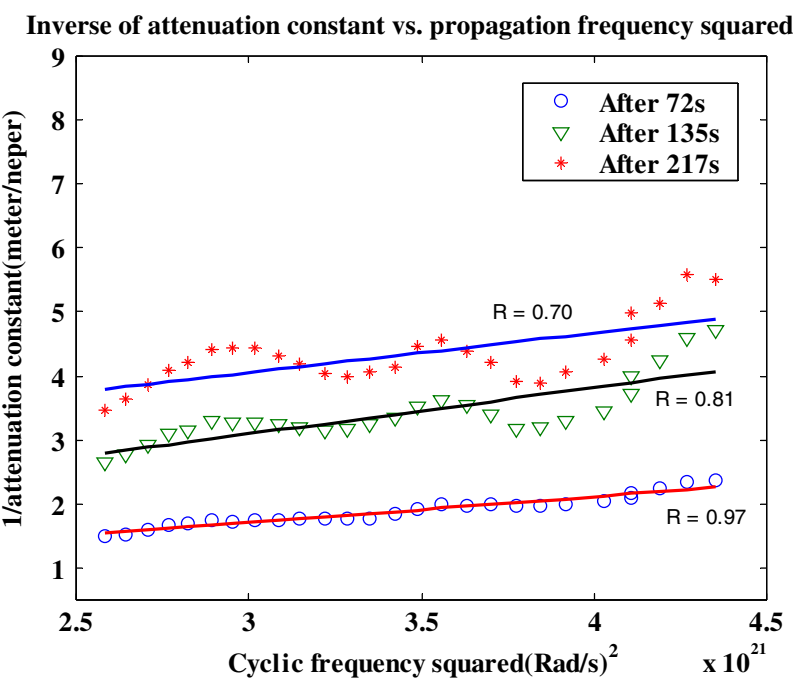

Figure 9. Variation of inverse of attenuation constant with propagation frequency squared.

and ordinate gave calculated electron density and collision frequency as $7.33 \times 10^{15} \mathrm{~m}^{-3}$ and $3.56 \times 10^{10} \mathrm{~s}^{-1}$. The gradient and ordinate for cooler flames at AS3 and AS4 gave electron density and collision frequency as $5.41 \times 10^{15} \mathrm{~m}^{-3}$ and $5.75 \times 10^{10} \mathrm{~s}^{-1}$ and $5.13 \times 10^{15} \mathrm{~m}^{-3}$ and $5.97 \times 10^{10} \mathrm{~s}^{-1}$ respectively.

The general behaviour of electron density in the combustion of pine litter, thus from AS1 to AS4, was that it decayed exponentially with time from a near maximum to a low value of $5.13 \times 10^{15} \mathrm{~m}^{-3}$ (see figure 10). The measured data fitted the exponential decay function of first order in the form: $N=N_{0}+A \exp \left(\frac{-x}{t}\right)$, where $N_{0}$ is near maximum electron concentration, $A$ and $x$ are flame dependent parameters. The variable $t$ is time.

Electron-neutral collision frequency was observed to increase rapidly with the decrease in flame temperature (see figure 11). The influx of cool ambient air nitrogen dilutes the combustion gas, which in effect increases neutral gas 


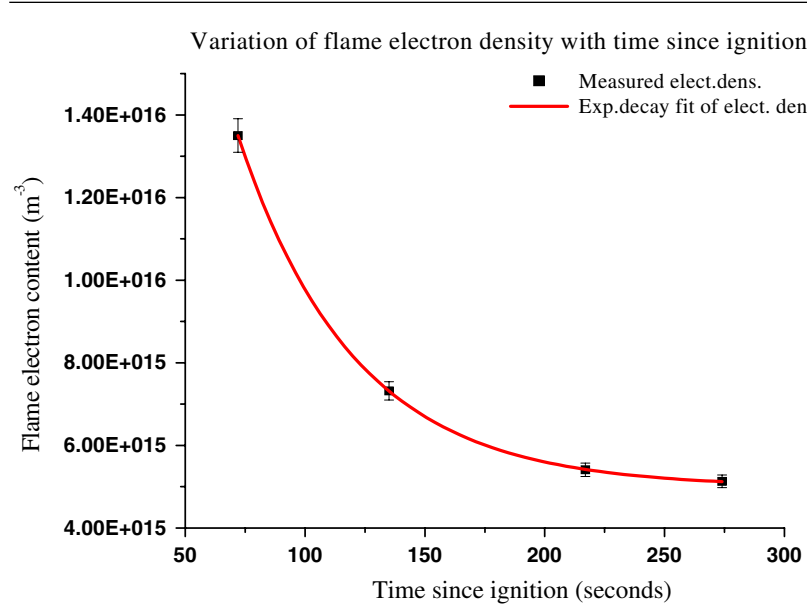

Figure 10. Variation of flame electron density with time after ignition.

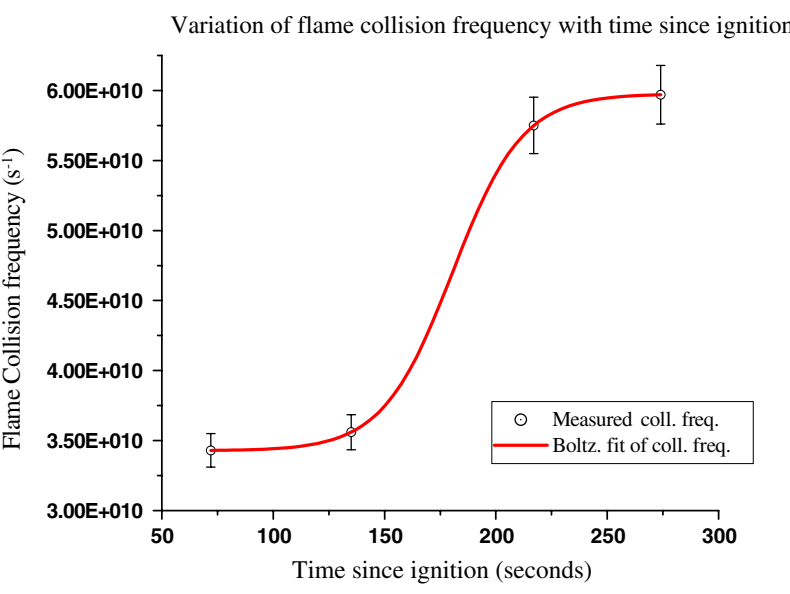

Figure 11. Variation of flame collision frequency with time after ignition.

concentration in the flame. This increases the rate of neutral collision with the available electrons in the flame.

6.3.1. Errors in electron density and collision frequency estimation. The electron density and collision frequency were determined by a graphical method. One of the ways to determine the errors in the parameters is to use the gradient and ordinate values and their associated errors, e.g. in [27]. When using this method, error in the electron density $(\Delta N)$ is given by the expression

$$
\Delta N=\frac{N}{2 \times \text { grad. } \times \text { ord. }}\left\{\frac{\Delta \text { ord. }}{\text { ord. }}+\frac{\Delta \text { grad. }}{\text { grad. }}\right\} .
$$

Similarly,

$$
\Delta \varphi_{\mathrm{eff}}=\frac{\varphi_{\mathrm{eff}}}{2 \times \operatorname{grad} \times \text { ord. }}\left\{\frac{\Delta \text { ord. }}{\text { ord. }}+\frac{\Delta \text { grad. }}{\text { grad. }}\right\},
$$

where $\Delta \operatorname{grad}$. is the difference between the best and the worst fit slopes and $\Delta$ ord. is the difference in the ordinate intercepts of the best and the worst fits. The errors are shown as error bars in figures 10 and 11 . They are in the range $3.7-4.3 \%$. The errors for the time coordinates in figures are too small to show. The errors introduced by the change in the dielectric properties of the thermal insulator were treated in a similar way as in [26].

\section{Conclusions}

The electron density for the pine fire with maximum radiant heat up to about $62 \mathrm{~kW} \mathrm{~m}^{-2}$ was in the range $(0.51-1.35) \times$ $10^{16} \mathrm{~m}^{-3}$. The electron densities were lower than those produced from non-vegetation fuel flames with much higher temperatures, e.g. Belcher and Sudden [2] and Koretzky and Kuo [1], who used a Meker type burner and plasma torches, respectively. However, electron concentration in the flame depends on the type of fuel as well as its temperature because it is this combination that produces ionizable particles.

The collision frequency of the flames was in the range $3.43-5.97 \times 10^{10} \mathrm{~s}^{-1}$. The range is lower than the electronneutral collision frequency at normal air temperature. The collision frequency is directly related to the neutral gas density and medium temperature. At ambient air temperatures the electron-neutral collision frequency is about $2.3 \times 10^{12} \mathrm{~s}^{-1}$ (see e.g. in [10]). For the pine litter fire, attenuation is, however, related in a complex way to both the electron density and the collision frequency, which are both related to electron content and flame temperature. From the experiment, $8-10.5 \mathrm{GHz}$ microwaves experienced relative attenuation in the range 1.64$5.68 \mathrm{~dB}$ when propagating in a line-of-sight mode through flame.

\section{Acknowledgments}

The authors would like to acknowledge the Department of Electrical Engineering of JCU for providing the equipment for s-parameter measurement. The work is supported by the Staff Development Office of the University of Botswana. It is partly supported by Emergency Management Australia under project no 60/2001.

\section{References}

[1] Koretzsky E and Kuo S P 1998 Characterization of atmospheric pressure plasma generated by a plasma torch array Phys. Plasmas $\mathbf{5} 3774-80$

[2] Belcher H and Sudden T M 1950 Studies on the ionisation produced by metallic salts in flames : II. Reactions governed by ionic equilibrium in coal-gas/air flames containing alkali metal salts Proc. R. Soc. Lond. A 202 17-39

[3] Williams D W, Adams J S, Batten J J, Whitty G F and Richardson G T 1970 Operation Euroka: An Australian Mass Fire Experiment Report 386, Defense Standards Laboratory, Maribyrnor Victoria, Australia

[4] Hata M and Shigeyuki D 1983 Propagation tests for $23 \mathrm{GHz}$ and $40 \mathrm{GHz}$ IEEE J. Sel. Areas Commun. $1658-73$

[5] Sorokin A, Vancassel X and Mirabel P 2002 Emission of ions and charged soot particle by aircraft engines Atmos. Chem. Phys. Discuss. 2 2045-74

[6] Latham D 1999 Space charge generated by wind tunnel fires Atmos. Res. 51 267-78

[7] Butler C J and Hayhurst A N 1998 Kinetics of gas-phase ionization of an alkali metal, $\mathrm{A}$, by the electron and proton transfer reactions: $\mathrm{A}+\mathrm{H}_{3} \mathrm{O}^{+} \rightarrow \mathrm{A}+. \mathrm{H}_{2} \mathrm{O}+\mathrm{H}$; $\mathrm{AOH}+\mathrm{AOH}_{2}+\mathrm{H}_{2} \mathrm{O}$ in fuel-rich flames at $1800-2250 \mathrm{~K}$ J. Chem. Soc. Faraday Trans. 98 2729-34

[8] Varadan V V, Jose K A and Varadan V K 2000 In situ microwave characterization of nonplanar dielectric objects IEEE Trans. Microw. Theory Tech. 48 388-94

[9] Vodacek A, Kremens R L, Fordham S C, VanGorden S C, Luisi D, Schott J R and Latham D J 2002 Remote optical 
detection of biomass burning using potassium emission signature Int. J. Remote Sens. 23 2721-6

[10] Akhtar K, Scharer E J, Tysk S M and Kho E 2003 Plasma interferometry at high pressures Rev. Sci. Instrum. 74 996-1001

[11] Smith H and Sudden T M 1952 Studies on ionisation produced by metallic salts in flames : III. Ionic equilibria in hydrogen/air flames containing alkali metal salts Proc. $R$. Soc. A 211 31-54

[12] Belcher H and Sudden T M 1950 Studies on ionisation produced by metallic salts in flames : I. Determination of collision frequency of electrons in coal gas-air flame Proc. R. Soc. A $201480-8$

[13] Shuler K E and Weber J 1954 A microwave investigation of the ionization of hydrogen-oxygen and acetylene-oxygen flames J. Chem. Phys. 22 491-502

[14] Sturgeon R E, Berman S S and Kashyaps S 1980 Microwave attenuation determination of electron concentration in graphite and tantalum tube electrothermal atomizers Anal. Chem. 52 1049-53

[15] Adler F P 1954 Measurement of conductivity of a jet flame J. Appl. Phys. 25 903-8

[16] Gangopadhyay P K, Lahiri-Dutt K and Saha K 2006 Application of remote sensing to identify coal fires in Raniganj Coalbelt India Int. J. Appl. Earth Obs. Geoinf. 8 188-95

[17] Sifakis N, Paronis D and Keramitsoglou I 2004 Combining AVHRR imagery with CORINE land cover data to observe forest fires and asses their consequences Int. J. Appl. Earth Obs. Geoinf. 5 263-74

[18] Radojevic M 2003 Chemistry of forest fires and regional haze with emphasis on Southeast Asia Pure Appl. Geophys. 12 $157-87$
[19] Okuno T, Sonoyama N, Hayashi J, Li C, Sathe C and Chiba T 2005 Primary release of alkali and alkaline earth metallic species during pyrolysis of pulverized biomass Energy Fuels 19 2164-71

[20] Alkemade M A 1979 Fundamentals of Analytical Flame Spectroscopy (Bristol: Hilger)

[21] Jensen A P, Frandsen F J, Dam-Johansen K and Sander B 2000 Experimental investigation of the transformation and release to gas phase of potassium and chlorine during straw pyrolysis conditions Energy Fuels 11 1026-32

[22] Dupuy J L, Marechal J and Morvan D 2003 Fires from a cylindrical forest fuel burner: combustion dynamics and flame properties Combust. Flame 135 65-76

[23] Frost L S 1961 Conductivity of seeded atmospheric pressure plasmas J. Appl. Phys. 32 2029-36

[24] Mphale K and Heron M L 2007 Plant alkali content and radio wave communication efficiency in high intensity savanna wildfires. J. Atmos. Sol. Terr. Phys. 69 471-84

[25] Vyskocil J and Musil J 1980 Microwave measurement of electron density and temperature in plasmas produced by a surfatron at atmospheric pressure J. Phys. D: Appl. Phys. 13 L25-8

[26] Varadan V V, Hollinger R D, Ghodgaonkar D K and Varadan V K 1991 Free-space broadband measurement of high temperature, complex dielectric properties at microwaves IEEE Trans. Instrum. Meas. 40 842-6

[27] Baird D C 1988 Experimentation: An Introduction to Measurement Theory and Experiment Design 2nd edn (Englewood Cliffs NJ: Prentice-Hall) 07632 137-50

[28] Boughriet A H, Legrand C and Chapoton A 1997 Noniterative stable transmission/reflection method for low-loss material complex permittivity determination IEEE Trans. Microw. Theory Tech. 45 52-6 